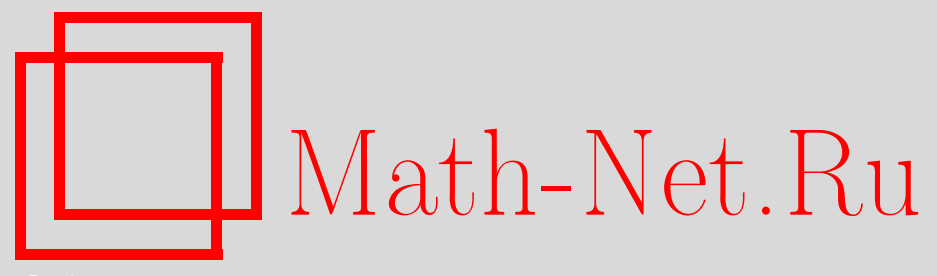

С. С. Марченков, $A$-замкнутые классы многозначной логики, содержащие константы, Дискрет. матем., 1998, том 10, выпуск $3,10-26$

DOI: https://doi.org/10.4213/dm436

Использование Общероссийского математического портала Math-Net.Ru подразумевает, что вы прочитали и согласны с пользовательским соглашением http://www.mathnet.ru/rus/agreement

Параметры загрузки:

IP: 18.207 .199 .55

26 апреля 2023 г., 18:04:00

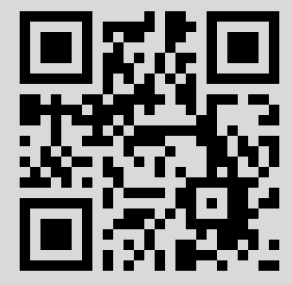




\title{
$A$-замкнутые классы многозначной логики, содержашие константы
}

(C) 1998 г. С. С. Марченков

\begin{abstract}
$A$-замыкание на множестве $P_{k}$ функций $k$-значной логики определяется как замыкание относительно операций суперпозиции и перехода к двойственным функциям для четных подстановок на множестве $E_{k}=\{0,1, \ldots, k-1\}$. При любом $k, k \geqslant 4$, описываются все $A$-замкнутые классы из $P_{k}$, содержащие константы. В качестве следствия получается описание всех $A$-замкнутых классов, содержащихся в классе Слупецкого, а также $A$-классификация симметрической полугруппы отображений множества $E_{k}$ в себя.

Работа выполнена при поддержке Российского фонда фундаментальных исследований, проект 97-01-00089.
\end{abstract}

\section{1. Введение}

В вопросах классификации функций многозначной логики наибольшее развитие получило направление, в котором классификации проводятся на основе операции суперпозиции и некоторых других близких операций. Ярким достижением этого направления явилась счетная классификация булевых функций, выполненная Постом [1-3] в 1921-1941 гг. Вместе с тем, как обнаружили Ю. И. Янов и А. А. Мучник [4], непосредственное обобщение результатов Поста на функции $k$-значной логики $(k \geqslant 3)$ невозможно : оно приводит к неэффективным континуальным классификациям.

В конце 70-х-начале 80-х годов появились результаты [5-9], которые дали основание предполагать, что эффективные (быть может, даже конечные) и нетривиальные классификации множеств $P_{k}$ функций $k$-значной логики можно получить, если наряду с операцией суперпозиции рассматривать операции перехода $\mathrm{k}$ двойственным функциям для подстановок из достаточно большой группы $G(G-$ классификации). Эта идея для полной симметрической группы $S_{k}$ была детально проработана Нгуен Ван Хоа [10-12] и автором [13-16]. Соответствующая классификация в $[13,14]$ была названа $S$-классификацией.

Оказалось, что $S$-классификация множества $P_{k}$ при любом $k, k \geqslant 3$, является конечной классификацией, число $S$-замкнутых классов в которой зависит от $k$ сверхэкспоненциальным образом. Каждый $S$-замкнутый класс имеет конечный базис по суперпозиции и (при наличии в нем селекторных функций) может быть определен как класс сохранения конечного набора отношений. 
Дальнейшие исследования $[15,17,18]$ по $G$-классификациям установили принципиальную возможность построения подобных конечных классификаций для менее широких групп $G$, которые удовлетворяют некоторым ограничениям на степень транзитивности. Вместе с тем опыт построения $S$-классификации показал, что в силу значительности объема сопутствующих построению определений, выкладок и доказательств провести в полной мере $G$-классификацию множеств $P_{k}$ возможно еще, по-видимому, только для знакопеременных подгрупп $A_{k}$ групп $S_{k}$. $G$-классификацию множества $P_{k}$ при $G=A_{k}$ мы называем $A$-классификацией.

Из [19] следует, что $A$-классификация множества $P_{3}$ континуальна. В $[15,18]$ доказано, что при любом $k, k \geqslant 5$, единственными $A$-предполными классами в $P_{k}$ являются класс $I_{k}$ идемпотентных функций и класс $S L P_{k}$ Слупецкого. При $k=4 \mathrm{k}$ этим двум классам необходимо добавить класс $K_{4}$ функций, самодвойственных относительно подстановок из четверной группы Клейна, и класс $L_{4}$ линейных функций (обычно [20] этот класс называют классом квазилинейных функций, однако для нас в данной работе более важным будет другое понятие квазилинейности [21]). Кроме того, в [15] установлено, что класс $K_{4}$ имеет ровно два $A$-предполных класса, $K_{4} \cap I_{4}$ и $K_{4} \cap L_{4}$.

Таким образом, дальнейшее построение $A$-классификации множеств $P_{k}, k \geqslant 4$, можно разбить на три части : описание $A$-замкнутых классов из $I_{k}$, из $S L P_{k}$ и из $L_{4}$. Аналогичные исследования, проведенные для $S$-классификации, позволяют высказать прдположение, что наиболее простой должна быть третья часть построения, а наиболее сложной, как в идейном, так и в техническом плане, первая.

Цель настоящей работы - дать описание всех $A$-замкнутых классов, содержащих константы. По аналогии с $S$-классификацией эти классы должны составлять наиболее массивное (по числу элементов) множество в $A$-классификации. Кроме того, привлечение небольших дополнительных сведений из теории групп позволяет получить описание всех $A$-замкнутых классов, лежащих в $S L P_{k}$, а также дать $A$ классификацию симметрической полугруппы отображений $k$-элементного множества в себя. Тем самым будет построена “половина" $A$-классификации множеств $P_{k}$.

\section{2. Основные понятия}

Пусть $k \geqslant 3, E_{k}=\{0,1, \ldots, k-1\}, P_{k}$ - множество всех функций $k$-значной логики, т.е. функций, отображающих декартовы степени множества $E_{k}$ в множество $E_{k}$. На множестве $P_{k}$ обычным образом определяется операция суперпозиции $[22,23]$. Если $M \subseteq P_{k}$, то через $[M]$ обозначается замыкание множества $M$ относительно операции суперпозиции. Множества вида $[M]$ называются замкнутыми классами.

Через $S_{k}$ обозначаем полную симметрическую группу подстановок на $E_{k}$, а через $A_{k}, e_{k}$ и $V_{4}$ - соответственно знакопеременную и единичную подгруппы в $S_{k}$ и четверную группу Клейна. Если $f\left(x_{1}, \ldots, x_{n}\right) \in P_{k}$ и $\pi \in S_{k}$, то функцию $\pi^{-1}\left(f\left(\pi\left(x_{1}\right), \ldots, \pi\left(x_{n}\right)\right)\right)$ называем двойственной к функции $f$ и обозначаем через $f^{\pi}$. Если $M \subseteq P_{k}$ и $G$ - подгруппа группы $S_{k}$, то $G$-замыканием множества $M$ (обозначение $[M]_{G}$ ) называем замыкание множества $M$ относительно операций суперпозиции и перехода к двойственным функциям для подстановок из группы $G$. Если $G=S_{k}$, то $G$-замыкание называем также $S$-замыканием, а если $G=A_{k}$, то $A$-замыканием.

Если $f \in P_{k}$, то через $\operatorname{Im} f$ обозначаем область значений функции $f$. Пусть 
$M \subseteq P_{k}, n-$ натуральное число и $1 \leqslant m \leqslant k$. Через $M^{n}$ обозначаем множество всех $n$-местных функций из $M$, а через $M^{(m)}$ множество всех функций $f$ из $M$, для которых $|\operatorname{Im} f| \leqslant m$, где $|N|$ - число элементов множества $N$. Пусть $M^{n(m)}=M^{n} \cap$ $M^{(m)}$. В частности, $P_{k}^{(k)}=P_{k}$ и $P_{k}^{1(k)}$ есть множество всех одноместных функций из $P_{k}$. Множество $P_{k}^{1(k)}$, рассматриваемое вместе с бинарной операцией композиции (суперпозиции), носит название симмерической полугруппы отображений на $E_{k}$.

Пусть $1 \leqslant m \leqslant k$. Подполугруппа $F$ полугруппы $P_{k}^{1(k)}$ называется $m$-транзитивной, если для любых $m$ попарно различных элементов $a_{1}, \ldots, a_{m}$ из $E_{k}$ и любых $m$ (не обязательно различных) элементов $b_{1}, \ldots, b_{m}$ из $E_{k}$ в полугруппе $F$ найдется такая функция $f$, что $f\left(a_{i}\right)=b_{i}$ для всех $i, 1 \leqslant i \leqslant m$.

Пусть $k_{1}, k_{2}, \ldots, k_{r}$ - такие натуральные числа, что $k_{1} \geqslant k_{2} \geqslant \ldots \geqslant k_{r}$ и $k=$ $k_{1}+\ldots+k_{r}$. Следуя [11], называем набор $\left(k_{1}, \ldots, k_{r}\right)$ разбиением числа $k$, а число $r$ рангом разбиения. На множестве всех разбиений числа $k$ вводим отношение $\preceq$ частичного порядка : для любых двух разбиений

$$
\lambda_{1}(k)=\left(k_{1}^{1}, \ldots, k_{r}^{1}\right), \quad \lambda_{2}(k)=\left(k_{1}^{2}, \ldots, k_{s}^{2}\right)
$$

отношение $\lambda_{1}(k) \preceq \lambda_{2}(k)$ выполняется тогда и только тогда, когда существует разбиение $\left(N_{1}, \ldots, N_{r}\right)$ множества $\{1,2, \ldots, s\}$ на непустые попарно не пересекающиеся подмножества такое, что при $1 \leqslant i \leqslant r$

$$
k_{i}^{1}=\sum_{j \in N_{i}} k_{j}^{2}
$$

Пусть $f \in P_{k}^{1(k)}$ и $\operatorname{Im} f=\left\{b_{1}, \ldots, b_{m}\right\}$, где $\left|f^{-1}\left(b_{1}\right)\right| \geqslant \ldots \geqslant\left|f^{-1}\left(b_{m}\right)\right|$. Набор $\left(\left|f^{-1}\left(b_{1}\right)\right|, \ldots,\left|f^{-1}\left(b_{m}\right)\right|\right)$ является разбиением числа $k$ и обозначается через $\lambda^{f}(k)$. Множества $f^{-1}\left(b_{1}\right), \ldots, f^{-1}\left(b_{m}\right)$ называются блоками функции $f$. Разбиение называется единичным, если каждый блок состоит из одного элемента. В дальнейшем рассматриваются только неединичные разбиения.

Для произвольного разбиения $\lambda(k)$ пусть $H(\lambda(k))$ (соответственно $\left.H_{0}(\lambda(k))\right)$ обозначает множество всех таких функций $f$ из $P_{k}^{1(k)}$, что $\lambda^{f}(k)=\lambda(k)$ (соответственно $\lambda^{f}(k)=\lambda(k)$ и $f(b)=b$ для любого $b$ из $\left.\operatorname{Im} f\right)$.

Если $\lambda(k)$ - разбиение числа $k$, то пусть

$$
F\langle\lambda(k)\rangle=\left\{f: \lambda^{f}(k) \preceq \lambda(k)\right\} .
$$

Если $\lambda_{1}(k), \ldots, \lambda_{s}(k)$ - разбиения числа $k$, то полагаем

$$
F\left\langle\lambda_{1}(k), \ldots, \lambda_{s}(k)\right\rangle=F\left\langle\lambda_{1}(k)\right\rangle \cup \ldots \cup F\left\langle\lambda_{s}(k)\right\rangle .
$$

Если $f \in H(\lambda(k))$ и $g \in P_{k}^{1}$, то $\lambda^{g f}(k) \preceq \lambda^{f}(k)$ и потому $g f \in F\langle\lambda(k)\rangle$. Кроме того, множество $F\langle\lambda(k)\rangle$ очевидным образом замкнуто относительно перехода к двойственным функциям. Отсюда следует, что для любых разбиений $\lambda_{1}(k), \ldots, \lambda_{s}(k)$ множество $F\left\langle\lambda_{1}(k), \ldots, \lambda_{s}(k)\right\rangle$ является $S$ - и $A$-замкнутым.

\section{3. $\quad$-классификация симметрической полугруппы $P_{k}^{1(k)}$}

Лемма 1. Пусть $k \geqslant 3, f_{1}, f_{2} \in P_{k}^{1(k-1)}, \lambda^{f_{1}}(k)=\lambda^{f_{2}}(k)$ и для любого а из $\operatorname{Im} f_{i}$ выполияется равенство $f_{i}(a)=a, i=1,2$. Тогда $\left[\left\{f_{1}\right\}\right]_{A}=\left[\left\{f_{2}\right\}\right]_{A}$. 
Доказательство. Пусть

$$
\begin{gathered}
\operatorname{Im} f_{1}=\left\{a_{1}, \ldots, a_{p}\right\}, \quad \operatorname{Im} f_{2}=\left\{b_{1}, \ldots, b_{p}\right\} \\
M_{j}=f_{1}^{-1}\left(a_{j}\right), \quad N_{j}=f_{2}^{-1}\left(b_{j}\right), \quad 1 \leqslant j \leqslant p \\
\left|M_{1}\right| \geqslant \ldots \geqslant\left|M_{p}\right|, \quad\left|N_{1}\right| \geqslant \ldots \geqslant\left|N_{p}\right| .
\end{gathered}
$$

Определим подстановку $\pi$ из $S_{k}$ условиями

$$
\pi\left(N_{j}\right)=M_{j}, \quad \pi\left(b_{j}\right)=a_{j}, \quad 1 \leqslant j \leqslant p .
$$

Тогда $f_{2}=f_{1}^{\pi}$. Поэтому если подстановка $\pi$ четна, то $f_{2} \in\left[\left\{f_{1}\right\}\right]_{A}$.

Предположим, что $\pi$ нечетна. Если $\left|N_{1}\right| \geqslant 3$, то выберем в множестве $N_{1}$ элементы $c, d$, отличные от $b_{1}$, и заменим нечетную подстановку $\pi$ четной подстановкой $\pi_{1}$, где

$$
\pi_{1}(c)=\pi(d), \quad \pi_{1}(d)=\pi(c), \quad \pi_{1}(b)=\pi(b), \quad b \in E_{k} \backslash\{c, d\} .
$$

Для подстановки $\pi_{1}$ также будет иметь место равенство $f_{2}=f_{1}^{\pi_{1}}$.

Если $\pi$ нечетна и $\left|N_{p-1}\right|=\left|N_{p}\right|=1$, то $\pi$ можно заменить четной подстановкой $\pi_{2}$, где

$$
\pi_{2}\left(b_{p-1}\right)=a_{p}, \quad \pi_{2}\left(b_{p}\right)=a_{p-1}, \quad \pi_{2}(b)=b, \quad b \in E_{k} \backslash\left\{b_{p-1}, b_{p}\right\} .
$$

Вновь будем иметь $f_{2}=f_{1}^{\pi_{2}}$.

Пусть $\pi$ нечетна, $\left|N_{1}\right|=\ldots=\left|N_{p-1}\right|=2$ и $p \geqslant 3$. Если $N_{1}=\left\{b_{1}, c_{1}\right\}$, то рассмотрим такую четную подстановку $\pi_{3}$, что

$$
\pi_{3}\left(b_{1}\right)=\pi\left(c_{1}\right), \quad \pi_{3}\left(c_{1}\right)=\pi\left(b_{1}\right), \quad \pi_{3}(b)=\pi(b), \quad b \in E_{k} \backslash\left\{b_{1}, c_{1}\right\},
$$

и положим $f_{3}=f_{1}^{\pi_{3}}$. Функция $f_{3}$ отличается от функции $f_{1}^{\pi}$ только тем, что на множестве $N_{1}$ принимает значение $c_{1}$, а не значение $b_{1}$. Определим далее четную подстановку $\pi_{4}$ с цикловым разложением $\left(b_{1} c_{1}\right)\left(b_{2} b_{3}\right)$ (остальные циклы разложения одноэлементны). Тогда будем иметь $f_{2}=f_{3}^{\pi_{4}} f_{3}$.

Остается рассмотреть случай, когда $k=4$ и $\left|N_{1}\right|=\left|N_{2}\right|=2$. Пусть для определенности $a_{1}=0, a_{2}=2, M_{1}=\{0,1\}, M_{2}=\{2,3\}$. Обозначим через $g_{1}, g_{2}$ функции, двойственные к $f_{1}$ относительно четных подстановок $(0)(123)$ и (012)(3) (см. рис. 1). Положим $g_{3}=g_{2} g_{1} f_{1}$ и $f_{4}=g_{3} f_{1} g_{3}$. Замечаем, что $f_{4}=f_{1}^{\pi_{5}}$, где $\pi_{5}$ - транспозиция элементов 0,1 . Поэтому если $\pi$ - нечетная подстановка, то функцию $f_{1}^{\pi}$ можно получить в виде $f_{4}^{\sigma}$, где $\sigma$ - такая четная подстановка, что $\pi=\pi_{5} \sigma$. Лемма доказана.

Лемма 2. Пусть $k \geqslant 3, \lambda(k)$ - неединичное разбиение числа $k$. Тогда

$$
[H(\lambda(k))]=\left[H_{0}(\lambda(k))\right] .
$$

Доказательство. Включение $\left[H_{0}(\lambda(k))\right] \subseteq[H(\lambda(k))]$ выполняется по определению. Установим обратное включение. Пусть

$$
f \in H(\lambda(k)), \quad \operatorname{Im} f=\left\{a_{1}, \ldots, a_{p}\right\}, \quad M_{i}=f^{-1}\left(a_{i}\right), \quad i=1, \ldots, p .
$$

Выберем в множестве $H_{0}(\lambda(k))$ такую функцию $g$, что она принимает $p$ значений и постоянна на каждом из множеств $M_{1}, \ldots, M_{p}$. Пусть $b_{1}=g\left(M_{1}\right), \ldots, b_{p}=g\left(M_{p}\right)$. Считая, что $g \neq f$, предположим по индукции, что

$$
b_{1}=a_{1}, \ldots, b_{j}=a_{j}, b_{j+1} \neq a_{j+1}, \quad 0 \leqslant j<p .
$$



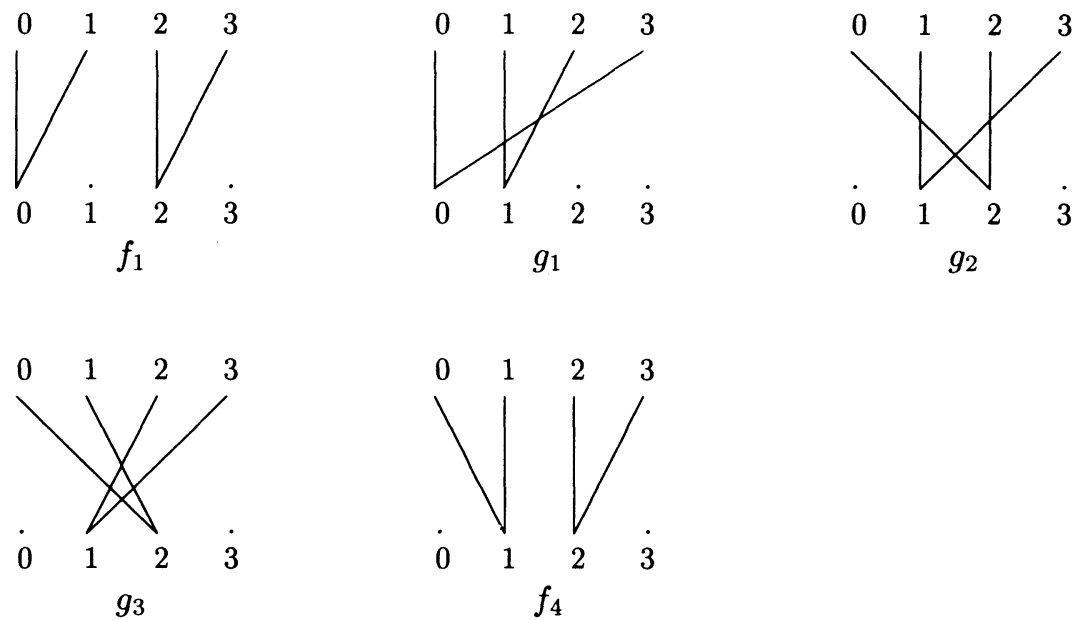

Puc. 1.

Далее рассмотрим две возможности.

Пусть $a_{j+1} \notin \operatorname{Im} g$. Пользуясь тем, что $p<k$, найдем в множестве $H_{0}(\lambda(k))$ такую функцию $h$, что $h\left(b_{j+1}\right)=h\left(a_{j+1}\right)=a_{j+1}$ и $h\left(b_{l}\right)=b_{l}$ для всех $l, l \neq j+1$. Полагая $g_{1}=h g$, получаем, что $g_{1}\left(M_{l}\right)=f\left(M_{l}\right)$ при $1 \leqslant l \leqslant j+1$ и $g_{1}\left(M_{l}\right)=g\left(M_{l}\right)$ при $j+1<l \leqslant p$. Таким образом, функция $g_{1}$ совпадает с функцией $f$ на множествах $M_{1}, \ldots, M_{j+1}$.

Предположим, что $a_{j+1} \in \operatorname{Im} g$ и, например, $b_{p}=g\left(M_{p}\right)=a_{j+1}$. Обозначим через $c$ какой-либо элемент из $E_{k} \backslash \operatorname{Im} g$. Выберем в множестве $H_{0}(\lambda(k))$ такую функцию $h$, что $h\left(b_{p}\right)=h(c)=c$ и $h\left(b_{l}\right)=b_{l}$ при $1 \leqslant l<p$. Тогда функция $h g$ возвращает нас к рассмотренной первой возможности. Лемма доказана.

Лемма 3. Пусть $k \geqslant 3, \lambda(k), \lambda^{\prime}(k)$ - такие неединичные разбиения числа $k$, что $\lambda^{\prime}(k) \preceq \lambda(k)$. Тогдa

$$
H\left(\lambda^{\prime}(k)\right) \subseteq[H(\lambda(k))]_{A}
$$

Доказателъство. Пусть

$$
f \in H(\lambda(k)), \quad \operatorname{Im} f=\left\{a_{1}, \ldots, a_{p}\right\}, \quad p \geqslant 2, \quad M_{i}=f^{-1}\left(a_{i}\right), \quad i=1, \ldots, p .
$$

Покажем, как из функции $f$ получить некоторую функцию $g$, у которой блоками являются, например, множества $M_{1} \cup M_{2}, M_{3}, \ldots, M_{p}$. С этой целью выберем в множестве $H(\lambda(k))$ такую функцию $f_{1}$, что $f_{1}\left(a_{1}\right)=a_{2}$ и $f_{1}\left(a_{i}\right)=a_{i}$ при $2 \leqslant i \leqslant p$. Это сделать можно, поскольку $p<k$. Полагаем $g=f_{1} f$. Далее применяем леммы 1 и 2. Лемма доказана.

Лемма 4. Пусть $k \geqslant 3, f \in P_{k}^{1(k-1)}$. Тогда

$$
[\{f\}]_{A}=\left\{g: g \in P_{k}^{1(k-1)}, \lambda^{g}(k) \preceq \lambda^{f}(k)\right\} .
$$


Доказательство. Следует воспользоваться леммами 1-3 и заметить, что в $[\{f\}]_{A}$ входят лишь такие функции $g$, для которых $\lambda^{g}(k) \preceq \lambda^{f}(k)$.

Из леммы 4 и $A$-замкнутости классов вида $F\left\langle\lambda_{1}(k), \ldots, \lambda_{s}(k)\right\rangle$ вытекает следующее утверждение.

Теорема 1. При любом $k, k \geqslant 3$, любой $A$-замкнутый класс функций из $P_{k}^{1(k-1)}$ можно представить в виде $F\left\langle\lambda_{1}(k), \ldots, \lambda_{s}(k)\right\rangle$, где разбиения $\lambda_{1}(k), \ldots, \lambda_{s}(k)$ попарно не сравнимы в смысле отношения с точностъю до перестановки разбиений в последователъности $\lambda_{1}(k), \ldots, \lambda_{s}(k)$.

Известно (см., например, [24]), что уравнение $G^{A_{k}}=G$, где $G$ - подгруппа группы $S_{k}$, имеет лишь решения $e_{k}, A_{k}, S_{k}$ и (при $k=4$ ) $V_{4}$. Иными словами, всякий $A$-замкнутый класс функций из $S_{k}$ совпадает с одним из классов $e_{k}, A_{k}, S_{k}$ или $V_{4}$. Кроме того, если $f \in P_{k}^{1(k-1)}$ и $g \in S_{k}$, то $\lambda^{f g}(k)=\lambda^{g f}(k)=\lambda^{f}(k)$. Поэтому, используя теорему 1 , приходим к следующему утверждению.

Теорема 2 (A-классификация симметрической полугруппы $\left.P_{k}^{1(k)}\right)$. При любом $k$, $k \geqslant 3$, любой $A$-замкнутый класс функций из $P_{k}^{1(k)}$ либо совпадает с одной из групп $e_{k}, A_{k}, S_{k}$ или $V_{4}$, либо представим в виде $F\left\langle\lambda_{1}(k), \ldots, \lambda_{s}(k)\right\rangle \cup G$, где разбиения $\lambda_{1}(k), \ldots, \lambda_{s}(k)$ попарно не сравнимы в смысле отношения $\preceq G \in\left\{\varnothing, e_{k}, A_{k}, S_{k}, V_{4}\right\}$.

\section{4. Класс Слупецкого}

Лемма 5. Пусть $k \geqslant 3, f \in P_{k}^{(k-1)} u|\operatorname{Im} f| \geqslant 2$. Тогда $[\{f\}]_{A}$ содержсит 2-транзитивную подполугруппу полугруппљ $P_{k}^{1(2)}$.

Доказательство. Положим $g_{1}(x)=f(x, \ldots, x)$. Так как $g_{1} \in P_{k}^{1(k-1)}$, согласно лемме 4 множество $\left[\left\{g_{1}\right\}\right]_{A}$ содержит все функции $h$, для которых $\lambda^{h}(k) \preceq \lambda^{g_{1}}(k)$. В частности, множеству $\left[\left\{g_{1}\right\}\right]_{A}$ принадлежат все функции-константы.

Из условия $|\operatorname{Im} f| \geqslant 2$ следует, что функция $f\left(x_{1}, \ldots, x_{n}\right)$ существенно зависит по крайней мере от одной переменной. Пусть это будет, например, переменная $x_{1}$. Тогда можно выбрать такие константы $c_{2}, \ldots, c_{n}$, что функция $g_{2}(x)=$ $f\left(x, c_{2}, \ldots, c_{n}\right)$ принимает не менее двух значений (в случае $n=1$ полагаем $g_{2}(x)=$ $f(x))$. В силу леммы 4 множеству $\left[\left\{g_{2}\right\}\right]_{A}$ принадлежат все функции $h$ такие, что $\lambda^{h}(k) \preceq \lambda^{g_{2}}(k)$. В частности, так как $\left|\operatorname{Im} g_{2}\right| \geqslant 2$, все указанные функции $h$, принимающие не более двух значений, образуют 2-транзитивную подполугруппу полугруппы $P_{k}^{1(2)}$. Лемма доказана.

Назовем функцию существенной, если она существенно зависит по крайней мере от двух переменных.

Лемма 6. Пусть $3 \leqslant l \leqslant k-1, f\left(x_{1}, \ldots, x_{n}\right)$ - существенная функция из $P_{k}^{(k-1)}$, принимающая l значений. Тогда $[\{f\}]_{A}=P_{k}^{(l)}$.

Доказательство. Докажем вначале по индукции, что $[\{f\}]_{A}$ содержит $l$-транзитивную подполугруппу полугруппы $P_{k}^{1(l)}$. Возьмем в качестве базиса индукции утверждение леммы 5 и предположим, что уже установлена принадлежность множеству $[\{f\}]_{A}$ некоторой $m$-транзитивной подполугруппы $F$ полугруппы $P_{k}^{1(m)}$, где 
$2 \leqslant m<l$. Пусть функция $f\left(x_{1}, \ldots, x_{n}\right)$ существенно зависит от переменной $x_{1}$. Согласно известной лемме о существенной функции (см. [23], лемма 4 из §4) найдутся такие $m+1$ наборов $\left(a_{1}^{1}, a_{2}^{1}, \ldots, a_{n}^{1}\right),\left(a_{1}^{2}, a_{2}^{1}, \ldots, a_{n}^{1}\right),\left(a_{1}^{1}, a_{2}^{3}, \ldots, a_{n}^{3}\right),\left(a_{1}^{4}, a_{2}^{4}, \ldots, a_{n}^{4}\right)$, $\ldots,\left(a_{1}^{m+1}, a_{2}^{m+1}, \ldots, a_{n}^{m+1}\right)$, что функция $f$ принимает на них $m+1$ значений. Так как последовательность $\left(a_{1}^{1}, a_{1}^{2}, a_{1}^{1}, a_{1}^{4}, \ldots, a_{1}^{m+1}\right)$ содержит не более $m$ различных значений, согласно лемме 4 в множество $[F]_{A}$ входит такая функция $f_{1}(x)$, которая при $x=0,1, \ldots, m$ принимает соответственно значения $a_{1}^{1}, a_{1}^{2}, a_{1}^{1}, a_{1}^{4}, \ldots, a_{1}^{m+1}$. Аналогичным образом устанавливаем, что в $[F]_{A}$ имеются такие функции $f_{2}, \ldots, f_{n}$, которые в точках $0,1, \ldots, m$ принимают соответствующие значения из последовательностей $\left(a_{2}^{1}, a_{2}^{1}, a_{2}^{3}, \ldots, a_{2}^{m+1}\right), \ldots,\left(a_{n}^{1}, a_{n}^{1}, a_{n}^{3}, \ldots, a_{n}^{m+1}\right)$. Следовательно, если определить $g(x)=f\left(f_{1}(x), f_{2}(x), \ldots, f_{n}(x)\right)$, то функция $g$ на множестве $\{0,1, \ldots, m\}$ примет $m+1$ значений. Так как $g \in P_{k}^{1(k-1)}$, в силу леммы 4 множество $[\{g\}]_{A}$ будет содержать некоторую $(m+1)$-транзитивную подполугруппу полугруппы $P_{k}^{1(m+1)}$.

Если, как и выше, выбрать $l$ наборов $\left(a_{1}^{1}, a_{2}^{1}, \ldots, a_{n}^{1}\right),\left(a_{1}^{2}, a_{2}^{1}, \ldots, a_{n}^{1}\right)$, $\left(a_{1}^{1}, a_{2}^{3}, \ldots, a_{n}^{3}\right),\left(a_{1}^{4}, a_{2}^{4}, \ldots, a_{n}^{4}\right), \ldots,\left(a_{1}^{l}, a_{2}^{l}, \ldots, a_{n}^{l}\right)$, на которых функция $f$ принимает $l$ различных значений $b_{1}, \ldots, b_{l}$, то можно применить теорему 4.4 .1 из [25]. Согласно этой теореме множество $[\{f\}]_{A}$ будет содержать, в частности, все функции из $P_{k}$, принимающие значения в множестве $\left\{b_{1}, \ldots, b_{l}\right\}$. Поскольку множество $[\{f\}]_{A}$ содержит также $l$-транзитивную подполугруппу полугруппы $P_{k}^{1(l)}$, приходим к соотношению $P_{k}^{(l)} \subseteq[\{f\}]_{A}$. Вместе с тем очевидно, что $[\{f\}]_{A} \subseteq P_{k}^{(l)}$. Лемма доказана.

Пусть $n \geqslant 2$. Квадратом называется любая четверка наборов вида

$$
\begin{aligned}
& \left(a_{1}, \ldots, a_{i-1}, b_{1}, a_{i+1}, \ldots, a_{j-1}, c_{1}, a_{j+1}, \ldots, a_{n}\right), \\
& \left(a_{1}, \ldots, a_{i-1}, b_{1}, a_{i+1}, \ldots, a_{j-1}, c_{2}, a_{j+1}, \ldots, a_{n}\right), \\
& \left(a_{1}, \ldots, a_{i-1}, b_{2}, a_{i+1}, \ldots, a_{j-1}, c_{1}, a_{j+1}, \ldots, a_{n}\right), \\
& \left(a_{1}, \ldots, a_{i-1}, b_{2}, a_{i+1}, \ldots, a_{j-1}, c_{2}, a_{j+1}, \ldots, a_{n}\right),
\end{aligned}
$$

где $b_{1} \neq b_{2}, c_{1} \neq c_{2}$.

Лемма 7. Пусть $k \geqslant 3, f\left(x_{1}, \ldots, x_{n}\right)$ - существенная функция из $P_{k}$, принимающая два значения, и существует квадрат, на котором функция $f$ принимает оба значения, причем одно из них в одной точке. Тогда $[\{f\}]_{A}=P_{k}^{(2)}$.

Доказательство. По леммам 4 и 5 множество $[\{f \cdot\}]_{A}$ содержит все константы. Считая, что квадрат из условия леммы имеет вид (1), подставим в функцию $f\left(x_{1}, \ldots, x_{n}\right)$ на места переменных $x_{1}, \ldots, x_{i-1}, x_{i+1}, \ldots, x_{j-1}, x_{j+1}, \ldots, x_{n}$ соответственно константы $a_{1}, \ldots, a_{i-1}, a_{i+1}, \ldots, a_{j-1}, a_{j+1}, \ldots, a_{n}$. Полученную функцию обозначим через $f_{1}\left(x_{i}, x_{j}\right)$. Функция $f_{1}$ принимает на квадрате $\left\{b_{1}, b_{2}\right\} \times\left\{c_{1}, c_{2}\right\}$ два значения, причем одно из них в одной точке. Поскольку в силу леммы 5 в $[\{f\}]_{A}$ содержится 2-транзитивная подполугруппа $F$ полугруппы $P_{k}^{1(2)}$, можно считать, что

$$
\operatorname{Im} f=\left\{b_{1}, b_{2}\right\}=\left\{c_{1}, c_{2}\right\}=\{0,1\}, \quad f_{1}(0,0)=1, \quad f_{1}(0,1)=f_{1}(1,0)=f_{1}(1,1)=0 .
$$

Тогда ограничение функции $f_{1}$ на множество $\{0,1\}$ совпадает с функцией $\bar{x}_{i} \bar{x}_{j}$, которая является шефферовой функцией в $P_{2}$. 
Покажем, что в $[\{f\}]_{A}$ входят все функции $g_{a}$ из $P_{k}^{1(2)}$ такие, что $g_{a}(a)=1$ и $g_{a}(x)=0$ при $x \neq a, a=0,1, \ldots, k-1$. Зафиксируем значение $a$ и, пользуясь 2транзитивностью полугруппы $F$, выберем в ней такую функцию $h$, что $h(a)=1$ и $\operatorname{Im} h=\{0,1\}$. Если $h(x)=0$ для всех $x, x \neq a$, то $g_{a}=h$. В противном случае пусть $b, c$ - такие элементы из $E_{k}$, что $b \neq a, h(b)=1$ и $h(c)=0$. Обозначим через $h_{1}$ функцию, двойственную к $h$ относительно четной подстановки $\pi=(a b c)$ (остальные циклы в цикловом разложении подстановки $\pi$ одноэлементны). Если $d=\pi^{-1}(1), e=\pi^{-1}(0)$, то $h_{1}(a)=h_{1}(c)=d$ и $h_{1}(b)=e$. Поскольку мы имеем в распоряжении 2-транзитивную полугруппу $F$, можно считать, что $d=1$ и $e=0$. Пользуясь шефферовостью ограничения $f_{1}$ на множество $\{0,1\}$, выберем в множестве $\left[\left\{f_{1}\right\}\right]$ такую функцию $f_{2}(x, y)$, чтобы ее ограничение на множество $\{0,1\}$ совпадало с булевой функцией $x y$. Полагая $h_{2}(x)=f_{2}\left(h(x), h_{1}(x)\right)$, видим, что $h_{2}(a)=1, h_{2}(b)=h_{2}(c)=0$ и $h_{2}(x) \leqslant h(x)$ при $x \notin\{a, b, c\}$. Таким образом, $h_{2}(a)=1$ и множество $h_{2}^{-1}(1)$ содержит по крайней мере на один элемент меньше, чем множество $h^{-1}(1)$. Если $h_{2} \neq g_{a}$, то продолжим этот процесс далее. В итоге получим функцию $g_{a}$.

Для завершения доказательства леммы остаєтся заметить,что всякую функцию $g\left(x_{1}, \ldots, x_{m}\right)$ из $P_{k}^{(2)}$, отличную от константы 0 и принимающую значения 0,1 , можно представить в виде аналога совершенной дизъюнктивной нормальной формы,

$$
g\left(x_{1}, \ldots, x_{m}\right)=\bigvee g_{a_{1}}\left(x_{1}\right) \& \ldots \& g_{a_{m}}\left(x_{m}\right),
$$

где через $\vee$ и \& обозначены подходящие функции из $\left[\left\{f_{1}\right\}\right]$, ограничения которых на $\{0,1\}$ являются соответствующими булевыми функциями дизъюнкции и конъюнкции.

Следуя [21], назовем функцию $f\left(x_{1}, \ldots, x_{n}\right)$ из $P_{k}$ квазилинйной, если для всякого $i, 1 \leqslant i \leqslant n$, и любых двух элементов $a, b$ из $E_{k}$ выполняется одно из двух условий : либо для любых $a_{1}, \ldots, a_{i-1}, a_{i+1}, \ldots, a_{n}$ из $E_{k}$

$$
f\left(a_{1}, \ldots, a_{i-1}, a, a_{i+1}, \ldots, a_{n}\right)=f\left(a_{1}, \ldots, a_{i-1}, b, a_{i+1}, \ldots, a_{n}\right),
$$

либо для любых $a_{1}, \ldots, a_{i-1}, a_{i+1}, \ldots, a_{n}$ из $E_{k}$

$$
f\left(a_{1}, \ldots, a_{i-1}, a, a_{i+1}, \ldots, a_{n}\right) \neq f\left(a_{1}, \ldots, a_{i-1}, b, a_{i+1}, \ldots, a_{n}\right) .
$$

Множество всех квазилинейных функций из $P_{k}$ обозначим через $Q L_{k}$, а множество $Q L_{k} \cap P_{k}^{(2)}$ - через $Q L_{k}^{(2)}$. Пользуясь определениями, убеждаемся в том, что множества $Q L_{k}$ и $Q L_{k}^{(2)}$ являются $A$-замкнутыми классами.

Как установлено в [21], всякую функцию $f\left(x_{1}, \ldots, x_{n}\right)$ из $Q L_{k}$ можно представить в виде

$$
f_{0}\left(f_{1}\left(x_{1}\right) \oplus \ldots \oplus f_{n}\left(x_{n}\right)\right)
$$

где $f_{0}, f_{1}, \ldots, f_{n} \in P_{k}^{1}$, а $\oplus$ обозначает сложение по модулю 2 . Из представления (2) следует, что при $n \geqslant 2$ для функций $f_{1}, \ldots, f_{n}$ можно считать выполненными равенства $\operatorname{Im} f_{1}=\ldots=\operatorname{Im} f_{n}=\{0,1\}$. Отсюда легко выводится, что

$$
Q L_{k}^{(2)}=\left[P_{k}^{1(2)} \cup\left\{f_{1}(x) \oplus f_{2}(y)\right\}\right]
$$


где $\operatorname{Im} f_{1}=\operatorname{Im} f_{2}=\{0,1\}$ и ограничения функций $f_{1}, f_{2}$ на множество $\{0,1\}$ являются тождественными функциями.

Дадим еще одно определение квазилинейной функции. Покажем, что $Q L_{k}$ совпадает с множеством $T$ всех функций $f\left(x_{1}, \ldots, x_{n}\right)$ таких, что либо $n=1$, либо $n \geqslant 2$ и функция $f$ на любом квадрате принимает не более двух значений, причем в случае двух значений каждое их них принимается в двух точках. Включение $Q L_{k} \subseteq T$ легко устанавливается, если воспользоваться представлением (2) для функций из класса $Q L_{k}$. Обратно, пусть $f\left(x_{1}, \ldots, x_{n}\right) \in T$ и пусть функция $f$ не удовлетворяет исходному определению квазилинейной функции. Полагая в нем для упрощения обозначений $i=1$, находим, что существуют такие элементы $a, b$ и наборы $\left(a_{2}, \ldots, a_{n}\right),\left(b_{2}, \ldots, b_{n}\right)$, что

$$
f\left(a, a_{2}, \ldots, a_{n}\right) \neq f\left(b, a_{2}, \ldots, a_{n}\right), \quad f\left(a, b_{2}, \ldots, b_{n}\right)=f\left(b, b_{2}, \ldots, b_{n}\right) .
$$

Из этих сооотношений следует, что наборы $\left(a_{2}, \ldots, a_{n}\right),\left(b_{2}, \ldots, b_{n}\right)$ различны. Пусть, например, $a_{j}=b_{j}$ при $j<m$ и $a_{j} \neq b_{j}$ при $m \leqslant j \leqslant n$. Рассматривая квадрат

$$
\begin{array}{ll}
\left(a, a_{2}, \ldots, a_{m-1}, a_{m}, b_{m+1}, \ldots, b_{n}\right), & \left(b, a_{2}, \ldots, a_{m-1}, a_{m}, b_{m+1}, \ldots, b_{n}\right), \\
\left(a, a_{2}, \ldots, a_{m-1}, b_{m}, b_{m+1}, \ldots, b_{n}\right), & \left(b, a_{2}, \ldots, a_{m-1}, b_{m}, b_{m+1}, \ldots, b_{n}\right)
\end{array}
$$

и используя равенство

$$
f\left(a, a_{2}, \ldots, a_{m-1}, b_{m}, \ldots, b_{n}\right)=f\left(b, a_{2}, \ldots, a_{m-1}, b_{m}, \ldots, b_{n}\right),
$$

заключаем, что должно выполняться также равенство

$$
f\left(a, a_{2}, \ldots ; a_{m}, b_{m+1}, \ldots, b_{n}\right)=f\left(b, a_{2}, \ldots, a_{m}, b_{m+1}, \ldots, b_{n}\right) .
$$

Далее аналогичным образом рассматриваем квадрат

$$
\begin{aligned}
\left(a, a_{2}, \ldots, a_{m}, a_{m+1}, b_{m+2}, \ldots, b_{n}\right), & \left(b, a_{2}, \ldots, a_{m}, a_{m+1}, b_{m+2}, \ldots, b_{n}\right), \\
\left(a, a_{2}, \ldots, a_{m}, b_{m+1}, \ldots, b_{n}\right), & \left(b, a_{2}, \ldots, a_{m}, b_{m+1}, \ldots, b_{n}\right)
\end{aligned}
$$

и получаем, что

$$
f\left(a, a_{2}, \ldots, a_{m+1}, b_{m+2}, \ldots, b_{n}\right)=f\left(b, a_{2}, \ldots, a_{m+1}, b_{m+2}, \ldots, b_{n}\right) .
$$

В итоге приходим к противоречию:

$$
f\left(a, a_{2}, \ldots, a_{n}\right)=f\left(b, a_{2}, \ldots, a_{n}\right) .
$$

Лемма 8. Пусть $k \geqslant 3, f\left(x_{1}, \ldots, x_{n}\right)$ - квазилинейная существенная функция вида (2), причем для некоторого $i, 1 \leqslant i \leqslant n$, в разбиении $\lambda^{f_{i}}(k)=\left(k_{1}, k_{2}\right)$ числа $k$ хотя бы одно из чисел $k_{1}, k_{2}$ нечетно. Тогда $[\{f\}]_{A}=Q L_{k}^{(2)}$.

Доказателъство. Без ограничения общности считаем, что функция $f\left(x_{1}, \ldots, x_{n}\right)$ существенно зависит от переменных $x_{1}, x_{2}$ и $\lambda^{f_{1}}(k)=\left(k_{1}, k_{2}\right)$, где одно из чисел $k_{1}, k_{2}$ нечетно. Согласно лемме 5 множество $[\{f\}]_{A}$ содержит 2-транзитивную подполугруппу $T$ полугруппы $P_{k}^{1(2)}$. Выберем в $T$ такие функции $h_{0}, h_{1}, h_{2}$, чтобы функция $h_{0}\left(f\left(h_{1}\left(x_{1}\right), h_{2}\left(x_{2}\right), 0, \ldots, 0\right)\right)$ имела представление вида $f_{1}^{\prime}\left(x_{1}\right) \oplus f_{2}^{\prime}\left(x_{2}\right)$, 
где $\operatorname{Im} f_{1}^{\prime}=\operatorname{Im} f_{2}^{\prime}=\{0,1\}$ и ограничения функций $f_{1}^{\prime}, f_{2}^{\prime}$ на множество $\{0,1\}$ являлись тождественными функциями. Тогда, как отмечалось выше, для доказательства леммы достаточно лишь установить, что $P_{k}^{1(2)} \subseteq[\{f\}]_{A}$.

Положим $h_{3}(x)=f(x, 0, \ldots, 0)$. Так как $h_{3}(x)=f_{0}\left(f_{1}(x) \oplus c\right)$, где $c=f_{2}(0) \oplus \ldots \oplus f_{n}(0)$, то $\lambda^{h_{3}}(k)=\left(k_{1}, k_{2}\right)$. Имея в распоряжении полугруппу $T$, можно предполагать, что $\operatorname{Im} h_{3}=\{0,1\}$. Пусть, например, $h_{3}^{-1}(1)=\left\{a_{1}, \ldots, a_{k_{1}}\right\}$, $h_{3}^{-1}(0)=\left\{b_{1}, \ldots, b_{k_{2}}\right\}$, где $k_{1}+k_{2}=k$ и $k_{1}$ нечетно. Покажем, как из функции $h_{3}$ получить такую функцию $g$, что $\operatorname{Im} g=\{0,1\}$ и $g$ принимает значение 1 только в одной точке.

Если $k_{1}=1$, то в качестве $g$ можно взять $h_{3}$. Пусть $k_{1} \geqslant 3$. Согласно лемме 4 в множество $\left[\left\{h_{3}\right\}\right]_{A}$ входит такая функция $h_{4}$, что

$$
\operatorname{Im} h_{4}=\{0,1\}, \quad h_{4}^{-1}(1)=\left\{a_{2}, \ldots, a_{k_{1}}, b_{1}\right\}, \quad h_{4}^{-1}(0)=\left\{a_{1}, b_{2}, \ldots, b_{k_{2}}\right\} .
$$

Положим $h_{5}(x)=f_{1}^{\prime}\left(h_{3}(x)\right) \oplus f_{2}^{\prime}\left(h_{4}(x)\right)$. Тогда

$$
\operatorname{Im} h_{5}=\{0,1\}, \quad h_{5}^{-1}(1)=\left\{a_{1}, b_{1}\right\}, \quad h_{5}^{-1}(0)=\left\{a_{2}, \ldots, a_{k_{1}}, b_{2}, \ldots, b_{k_{2}}\right\} .
$$

Выберем согласно лемме 4 в множестве $\left[\left\{h_{5}\right\}\right]_{A}$ такую функцию $h_{6}$, что

$$
\operatorname{Im} h_{6}=\{0,1\}, \quad h_{6}^{-1}(1)=\left\{a_{k_{1}-1}, a_{k_{1}}\right\}, \quad h_{6}^{-1}(0)=\left\{a_{1}, \ldots, a_{k_{1}-2}, b_{1}, \ldots, b_{k_{2}}\right\} .
$$

Определяя далее $h_{7}(x)=f_{1}^{\prime}\left(h_{3}(x)\right) \oplus f_{2}^{\prime}\left(h_{6}(x)\right)$, видим, что функция $h_{7}$ принимает значение 1 только в точках $a_{1}, \ldots, a_{k_{1}-2}$. Если $k_{1} \neq 3$, то описанный процесс следует продолжить. В итоге получим функцию $g$.

В силу леммы 4 множество $[\{g\}]_{A}$ содержит функции $g_{0}, g_{1}, \ldots, g_{k-1}$, такие, что $g_{j}(j)=1$ и $g_{j}(x)=0$ при $x \neq j, j=0,1, \ldots, k-1$. Пусть теперь $h-$ функция из $P_{k}^{1(2)}$, принимающая лишь значения 0,1 и отличная от константы 0 . Пусть $h^{-1}(1)=$ $\left\{a_{1}, \ldots, a_{s}\right\}$. Тогда

$$
h(x)=g_{a_{1}}(x) \oplus \ldots \oplus g_{a_{s}}(x) .
$$

Так как функции $g_{0}, \ldots, g_{k-1}$ принимают лишь значения 0,1 , в качестве функции $\oplus$ в приведенном равенстве можно использовать функцию $f_{1}^{\prime}(x) \oplus f_{2}^{\prime}(y)$. Для завершения доказательства леммы остается еще раз обратиться к лемме 4.

Пусть $k$ четно. Обозначим через $P_{k}^{1(2)+}$ множество всех таких функций $f$ из $P_{k}^{1(2)}$, что либо $f-$ константа, либо $\lambda^{f}(k)=\left(k_{1}, k_{2}\right)$, где оба числа $k_{1}, k_{2}$ четны. Пусть $Q L_{k}^{(2)+}$ - множество всех таких функций $f\left(x_{1}, \ldots, x_{n}\right)$ из $Q L_{k}^{(2)}$, у которых в представлении (2) все функции $f_{1}, \ldots, f_{n}$ принадлежат множеству $P_{k}^{1(2)+}$.

Нетрудно убедиться в том, что класс $Q L_{k}^{(2)+}$ является $A$-замкнутым.

Лемма 9. Пусть $f\left(x_{1}, \ldots, x_{n}\right)$ - существенная функция из множества $Q L_{k}^{(2)+}$. Тогда $[\{f\}]_{A}=Q L_{k}^{(2)+}$.

Доказательство. Из определения класса $Q L_{k}^{(2)+}$ следует, что $Q L_{k}^{(2)+}$ порождается множеством $P_{k}^{1(2)+}$ и любой функцией (из класса $Q L_{k}^{(2)+}$ ) вида $f_{1}^{\prime}(x) \oplus f_{2}^{\prime}(y)$, где $\operatorname{Im} f_{1}^{\prime}=\operatorname{Im} f_{2}^{\prime}=\{0,1\}$ и ограничения функций $f_{1}^{\prime}, f_{2}^{\prime}$ на множество $\{0,1\}$ являются тождественными функциями. Как и в лемме 8 , из функции $f$ получаем функции $f_{1}^{\prime}(x) \oplus f_{2}^{\prime}(y)$ и $h_{5}$, где $\operatorname{Im} h_{5}=\{0,1\}$ и функция $h_{5}$ принимает значение 1 только в 
двух точках. Далее замечаем, что всякую функцию из $P_{k}^{1(2)+}$, принимающую лишь значения 0,1 , можно получить путем сложения по модулю 2 (т.е. с помощью функции $\left.f_{1}^{\prime}(x) \oplus f_{2}^{\prime}(y)\right)$ подходящих функций из $\left[\left\{h_{5}\right\}\right]_{A}$, которые принимают значение 1 в двух точках. Лемма доказана.

Из лемм 6-9 и определений квазилинейных функций вытекает следующее утверждение.

Теорема 3. Пусть $k \geqslant 3, f-$ существенная функция из $P_{k}^{(k-1)}$. Тогда $[\{f\}]_{A}$ совпадает с одним из классов $P_{k}^{(k-1)}, \ldots, P_{k}^{(2)}, Q L_{k}^{(2)}$ или (при четном $k$ ) $Q L_{k}^{(2)+}$.

Класс $S L P_{k}$ Слупецкого состоит из всех функций, существенно зависящих не более чем от одной переменной, и всех функций, принимающих не более $k-1$ значений.

Теорема 4. При любом $k, k \geqslant 3$, любой $A$-замкнутый класс функций из $S L P_{k}$ совпадает с одним из следующих классов:

(1) $e_{k}, A_{k}, S_{k}$ uлu $V_{4}$

(2) $F\left\langle\lambda_{1}(k), \ldots, \lambda_{s}(k)\right\rangle$;

(3) $F\left\langle\lambda_{1}(k), \ldots, \lambda_{s}(k)\right\rangle \cup G$;

(4) $P_{k}^{(k-1)}, \ldots, P_{k}^{(2)}, Q L_{k}^{(2)}, Q L_{k}^{(2)+}$;

(5) $P \cup G$;

(6) $P \cup F\left\langle\lambda_{1}(k) \ldots, \lambda_{s}(k)\right\rangle$;

(7) $P \cup F\left\langle\lambda_{1}(k), \ldots, \lambda_{s}(k)\right\rangle \cup G$,

гдe

$$
G \in\left\{e_{k}, A_{k}, S_{k}, V_{4}\right\}, \quad P \in\left\{P_{k}^{(k-1)}, \ldots, P_{k}^{(2)}, Q L_{k}^{(2)}, Q L_{k}^{(2)+}\right\}
$$

и разбиения $\lambda_{1}(k), \ldots, \lambda_{s}(k)$ попарно не сравнимы в смысле отношения $\preceq$. Кроме того, в пn. 6,7 выполняются следующие дополнителъные условия. Если $P=P_{k}^{(l)}$, $2 \leqslant l \leqslant k-2$, то ранг каждого из разбиений $\lambda_{1}(k), \ldots, \lambda_{s}(k)$ больше $l$; если $P=$ $Q L_{k}^{(2)}$, то ранг кажсдого из разбиений $\lambda_{1}(k), \ldots, \lambda_{s}(k)$ болъше 2 ; если $P=Q L_{k}^{(2)+}$, то ранг каждого из разбиений $\lambda_{1}(k), \ldots, \lambda_{s}(k)$ больше 2 и каждый элемент любого разбиения четный.

Доказательство. Пусть $R$ - произвольный $A$-замкнутый класс функций из $S L P_{k}$. Если $R$ не содержит существенных функций, то его можно рассматривать как подмножество множества $P_{k}^{1(k)}$. Применяя теорему 2 , приходим к первым трем пунктам теоремы 4 . Предположим, что $R$ содержит существенную функцию. Тогда в соответствии с теоремой 3 класс $R$ содержит целиком один из классов $P_{k}^{(k-1)}, \ldots, P_{k}^{(2)}$, $Q L_{k}^{(2)}, Q L_{k}^{(2)+}$. Имеют место строгие включения

$$
P_{k}^{(k-1)} \supset \ldots \supset P_{k}^{(2)} \supset Q L_{k}^{(2)} \supset Q L_{k}^{(2)+}
$$

(последнее включение имеет место при четном $k$ ), поэтому пусть $P$ - максимальный из классов $P_{k}^{(k-1)}, \ldots, P_{k}^{(2)}, Q L_{k}^{(2)}, Q L_{k}^{(2)+}$, содержащихся в $R$. Если $R=P$, 
Таблица 1.

\begin{tabular}{|c|llll|}
\hline+ & 0 & 1 & 2 & 3 \\
\hline 0 & 0 & 1 & 2 & 3 \\
1 & 1 & 0 & 3 & 2 \\
2 & 2 & 3 & 0 & 1 \\
3 & 3 & 2 & 1 & 0 \\
\hline
\end{tabular}

Таблица 2.

\begin{tabular}{|c|lllll|}
\hline$\times$ & 0 & 1 & 2 & 3 \\
\hline 0 & 0 & 0 & 0 & 0 \\
1 & 0 & 1 & 2 & 3 \\
2 & 0 & 2 & 3 & 1 \\
3 & 0 & 3 & 1 & 2 \\
\hline
\end{tabular}

то получаем п. 4 теоремы 4 . Если же $R \neq P$, то множество $R \backslash P$ состоит только из функций, которые существенно зависят от одной переменной. В случае, когда все функции из $R \backslash P$ с точностью до несущественных переменных являются подстановками, мы с использованием теоремы 2 приходим к равенству $R=P \cup G$, где $G \in\left\{e_{k}, A_{k}, S_{k}, V_{4}\right\}$. Пусть, напротив, множество $R \backslash P$ не содержит ни одной подстановки. Тогда согласно теореме 2 множество всех одноместных функций из $R$ можно представить в виде $F\left\langle\lambda_{1}(k), \ldots, \lambda_{s}(k)\right\rangle$, где разбиения $\lambda_{1}(k), \ldots, \lambda_{s}(k)$ попарно не сравнимы в смысле отношения $\preceq$. Если $P=P_{k}^{(l)}$, то $P_{k}^{1(l)} \subset R$ и потому из последовательности $\lambda_{1}(k), \ldots, \lambda_{s}(k)$ можно удалить все разбиения, ранг которых не превосходит $l$. Аналогично поступаем при $P=Q L_{k}^{(2)}$, поскольку в этом случае $P_{k}^{1(2)} \subset R$. Пусть $k$ четно и $P=Q L_{k}^{(2)+}$. Если для некоторого $i, 1 \leqslant i \leqslant s$, один из элементов разбиения $\lambda_{i}(k)$ нечетен, то согласно рассуждениям из леммы 8 класс $R$ будет содержать все множество $Q L_{k}^{(2)}$, что невозможно в силу выбора класса $P$. Как и случае классов $P_{k}^{(l)}$, последовательность $\lambda_{1}(k), \ldots, \lambda_{s}(k)$ можно считать свободной от разбиений, ранг которых равен 2.

Если, наконец, множество $R \backslash P$ содержит элементы каждого из множеств $S_{k}$, $P_{k}^{1(k-1)}$, то объединяем рассуждения, которые использовались при рассмотрении пп. 5, 6. Теорема доказана.

Замечание 1. Сравнение с [11] показывает, что совокупность $A$-замкнутых классов, лежащих в $S L P_{k}$, полностью совпадает с совокупностью $S$-замкнутых классов из $S L P_{k}$.

\section{5. Класс $L_{4}$ линейных функций}

Как отмечалось во введении, при $k \geqslant 5$ единственными $A$-предполными классами в $P_{k}$ являются классы $I_{k}$ и $S L P_{k}$. При $k=4$ к этим классам добавляются классы $K_{4}$ и $L_{4}$. Классы $I_{4}$ и $K_{4}$ не содержат констант. Поэтому при $k \geqslant 5$ множество всех $A$ замкнутых классов из $P_{k}$, содержащих константы, описывается пп. 2-7 теоремы 4. При $k=4$ необходимо дополнительно исследовать $A$-замкнутые классы линейных функций, которые содержат константы и не входят в класс $S L P_{4}$.

Класс $L_{4}$ можно определить [20] как класс всех функций из $P_{4}$, которые сохраняют отношение $x_{1}+x_{2}=x_{3}+x_{4}$, где + обозначает сложение в поле Галуа $G F(4)$ и задается табл. 1. Несложные рассуждения показывают, что произвольную функцию из класса $L_{4}$ от $n$ переменных можно представить в виде

$$
a_{1} x_{1}^{2}+b_{1} x_{1}+\ldots+a_{n} x_{n}^{2}+b_{n} x_{n}+c
$$


где $a_{1}, b_{1}, \ldots, a_{n}, b_{n}, c \in E_{4}$, а умножение в поле Галуа $G F(4)$ определяется табл. 2. Положим

$$
\begin{aligned}
L_{4} A_{4} & =\left\{b_{1} x_{1}+\ldots+b_{n} x_{x}+c: b_{1}, \ldots, b_{n}, c \in E_{4}\right\} \\
L_{4} V_{4} & =\left\{b_{1} x_{1}+\ldots+b_{n} x_{n}+c: b_{1}, \ldots, b_{n} \in E_{2}, c \in E_{4}\right\} .
\end{aligned}
$$

Легко проверить, что

$$
\begin{aligned}
& V_{4}=\left\{x+c: c \in E_{4}\right\}, \quad A_{4}=\left\{b x+c: b, c \in E_{4}, b \neq 0\right\}, \\
& S_{4}=\left\{a x^{2}+c, b x+c: a, b, c \in E_{4}, a \neq 0, b \neq 0\right\} .
\end{aligned}
$$

Отсюда, в частности, вытекает, что классы $L_{4} A_{4}, L_{4} V_{4}$ являются $A$-замкнутыми. Отметим еще, что если $a \neq 0, b \neq 0$, то функция $a x^{2}+b x+c$ принимает два значения, причем каждое значение в двух точках.

Лемма 10. Пусть $f\left(x_{1}, \ldots, x_{n}\right)$ - существенная функция из $L_{4}$, принимающая 4 значения. Тогда $[\{0, f\}]_{A}$ совпадает с одним из классов $L_{4}, L_{4} A_{4}, L_{4} V_{4}$.

Доказательство. Будем предполагать, что функция $f\left(x_{1}, \ldots, x_{n}\right)$ представлена в виде (3), причем все переменные $x_{1}, \ldots, x_{n}$ являются существенными. Это означает, что $n \geqslant 2$ и в каждой паре $\left(a_{i}, b_{i}\right), i=1, \ldots, n$, хотя бы один из коэффициентов отличен от 0 .

Пусть сначала $a_{1} \neq 0$ и $b_{1}=0$. Подставляя в функцию $f\left(x_{1}, \ldots, x_{n}\right)$ константу 0 вместо переменных $x_{3}, \ldots, x_{n}$, получаем функцию

$$
f_{1}\left(x_{1}, x_{2}\right)=a_{1} x_{1}^{2}+a_{2} x_{2}^{2}+b_{2} x_{2}+c .
$$

Так как $f_{1}\left(x_{1}, 0\right)$ есть нечетная подстановка $a_{1} x_{1}^{2}+c$, то

$$
\left[\left\{f_{1}\left(x_{1}, 0\right)\right\}\right]_{A}=S_{4} \text {. }
$$

Если $a_{2} b_{2}=0$ (то есть функция $a_{2} x_{2}^{2}+b_{2} x_{2}$ также является подстановкой), то выберем в $S_{4}$ подстановки $h_{1}, h_{2}$, обратные к подстановкам $a_{1} x_{1}^{2}$ и $a_{2} x_{2}^{2}+b_{2} x_{2}+c$. Тогда

$$
f_{1}\left(h_{1}\left(x_{1}\right), h_{2}\left(x_{2}\right)\right)=x_{1}+x_{2} .
$$

Очевидно, что функция $x_{1}+x_{2}$ вместе с множеством $S_{4}$ образует полную в $L_{4}$ систему.

Допустим, что $a_{2} b_{2} \neq 0$. Тогда функция $a_{2} x_{2}^{2}+b_{2} x_{2}+c$ принимает ровно 2 значения, причем каждое значение в двух точках. Выберем в группе $S_{4}$ такие подстановки $h_{2}, h_{3}$, чтобы выполнялось равенство

$$
h_{3}\left(a_{2} h_{2}^{2}(x)+b_{2} h_{2}(x)+c\right)=2 x^{2}+3 x
$$

(функция $2 x^{2}+3 x$ принимает значение 0 при $x=0,2$ и значение 1 при $x=1,3$ ). Так как группа $A_{4} 2$-транзитивна, подстановку $h_{3}$ можно считать четной. Иными словами, можно предполагать, что $h_{3}(x)=d x+e$, где $d \neq 0$. Найдем далее в группе $S_{4}$ такую подстановку $h_{1}$, что выполняется равенство $d a_{1} h_{1}^{2}(x)=x$. Положим

$$
f_{2}\left(x_{1}, x_{2}\right)=h_{3}\left(f_{1}\left(h_{1}\left(x_{1}\right), h_{2}\left(x_{2}\right)\right)\right) .
$$


Тогда

$$
f_{2}\left(x_{1}, x_{2}\right)=d a_{1} h_{1}^{2}\left(x_{1}\right)+d\left(a_{2} h_{2}^{2}\left(x_{2}\right)+b_{2} h_{2}\left(x_{2}\right)+c\right)+e=x_{1}+2 x_{2}^{2}+3 x_{2} .
$$

Аналогичным образом получаем функцию

$$
f_{3}\left(x_{1}, x_{2}\right)=x_{1}+2 x_{2}^{2}+2 x_{2} .
$$

Теперь

$$
f_{2}\left(f_{3}\left(x_{1}, x_{2}\right), x_{2}\right)=x_{1}+x_{2} .
$$

Предположим, что $a_{1}=0$ и $b_{1} \neq 0$. Будем также считать, что для любого $i$, $2 \leqslant i \leqslant n$, либо $a_{i}=0$, либо $a_{i} b_{i} \neq 0$. Рассмотрим сначала случай, когда для некоторого $i, 2 \leqslant i \leqslant n$, выполняется неравенство $a_{i} b_{i} \neq 0$. Пусть, например, $i=2$. Как и выше, из функции $f\left(x_{1}, \ldots, x_{n}\right)$ подстановкой константы 0 образуем функцию

$$
f_{1}\left(x_{1}, x_{2}\right)=b_{1} x_{1}+a_{2} x_{2}^{2}+b_{2} x_{2}+c .
$$

Согласно лемме 4 множество $\left[\left\{f_{1}\left(0, x_{2}\right)\right\}\right]_{A}$ содержит все функции $h$ такие, что $\lambda^{h}(4)=(2,2)$. Выберем поэтому в $\left[\left\{f_{1}\left(0, x_{2}\right)\right\}\right]_{A}$ такую функцию $h_{1}(x)=d x^{2}+e x$, $d e \neq 0$, чтобы выполнялись соотношения

$$
b_{1} d+a_{2} \neq 0, \quad b_{1} e+b_{2}=0
$$

Тогда

$$
f_{1}\left(h_{1}(x), x\right)=\left(b_{1} d+a_{2}\right) x^{2}+c .
$$

Следовательно, функция $f_{1}\left(f_{1}\left(h_{1}\left(x_{1}\right), x_{1}\right), x_{2}\right)$ удовлетворяет условиям рассмотренного выше случая $a_{1} \neq 0, b_{1}=0$.

Предположим, что $a_{2}=\ldots=a_{n}=0$. Если $b_{1}=\ldots=b_{n}=1$, то из функции $f\left(x_{1}, \ldots, x_{n}\right)$ подстановкой константы 0 получаем функцию

$$
f_{1}\left(x_{1}, x_{2}\right)=x_{1}+x_{2}+c .
$$

Так как по условию леммы мы располагаем всеми константами, подстановкой подходящей константы из функции $f_{1}\left(x_{1}, x_{2}\right)$ можно получить функцию $x_{1}-c$. Функция

$$
f_{1}\left(x_{1}, x_{2}\right)-c=x_{1}+x_{2}
$$

вместе с контантами порождает класс $L_{4} V_{4}$. Поскольку, очевидно, $f \in L_{4} V_{4}$, в этом случае

$$
[\{0, f\}]_{A}=L_{4} V_{4} \text {. }
$$

Допустим, что один из коэффициентов $b_{1}, \ldots, b_{n}$, например $b_{1}$, отличен от 1 . Тогда

$$
f\left(x_{1}, 0, \ldots, 0\right)=b_{1} x_{1}+c
$$

есть четная подстановка, не входящая в $V_{4}$. Значит,

$$
\left[\left\{b_{1} x_{1}+c\right\}\right]_{A}=A_{4} \text {. }
$$

Нетрудно видеть, что в этом случае

$$
[\{0, f\}]_{A}=L_{4} A_{4}
$$




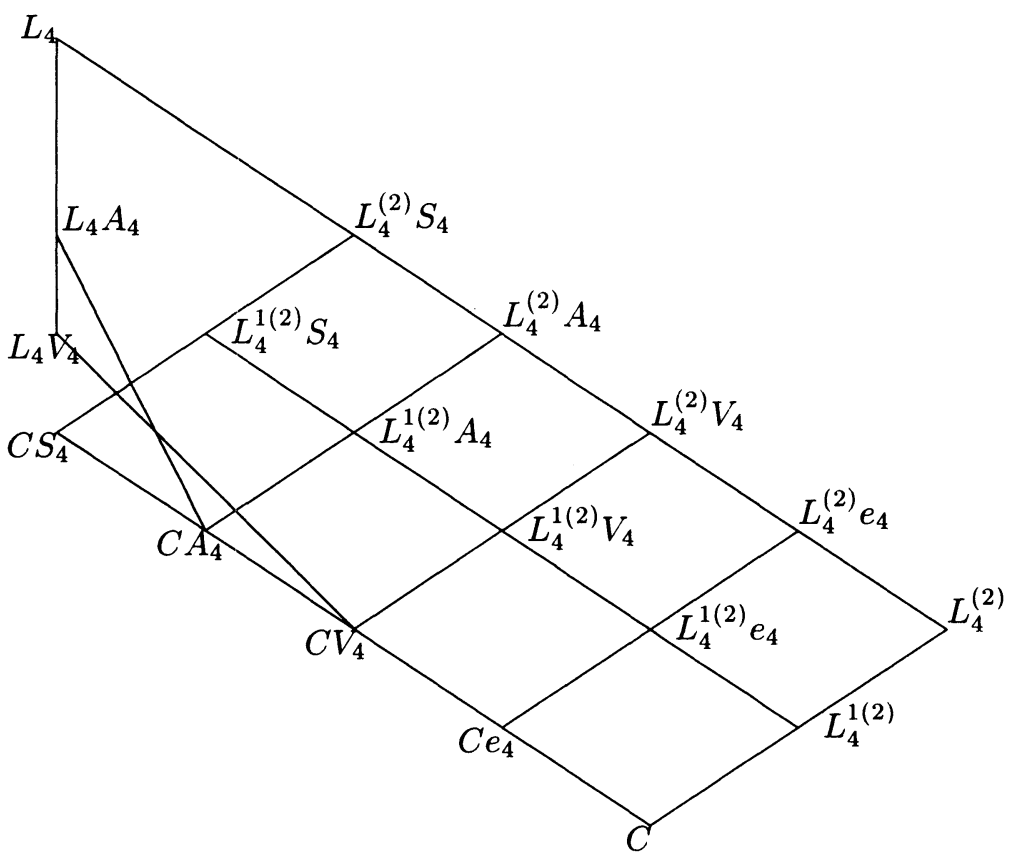

Рис. 2.

Предположим, наконец, что $a_{i} b_{i} \neq 0$ для всех $i, 1 \leqslant i \leqslant n$. Так как функция $f$ принимает 4 значения, области значений функций $a_{1} x_{1}^{2}+b_{1} x_{1}, \ldots, a_{n} x_{n}^{2}+b_{n} x_{n}$ не могут быть все одинаковыми. Пусть, например, $\operatorname{Im}\left(a_{1} x_{1}^{2}+b_{1} x_{1}\right) \neq \operatorname{Im}\left(a_{2} x_{2}^{2}+b_{2} x_{2}\right)$. Очевидно, что множество $[\{0, f\}]_{A}$ содержит все функции $h(x)$ такие, что $\lambda^{h}(4)=(2,2)$. Выберем поэтому функцию $h_{2}\left(x_{2}\right)$ из $[\{0, f\}]_{A}$ с таким расчетом, чтобы функция $a_{2} h_{2}^{2}\left(x_{2}\right)+b_{2} h_{2}\left(x_{2}\right)$ имела два блока, не совпадающие с блоками функции $a_{1} x_{1}^{2}+b_{1} x_{1}$. Можно проверить, что в этом случае $a_{1} x_{2}^{2}+b_{1} x_{2}+a_{2} h_{2}^{2}\left(x_{2}\right)+b_{2} h_{2}\left(x_{2}\right)$ есть подстановка. Следовательно, при $n \geqslant 3$ согласно рассмотренным выше случаям мы получим $[\{0, f\}]_{A}=L_{4}$, если вместо функции $f$ возьмем $f\left(x_{2}, h_{2}\left(x_{2}\right), x_{3}, \ldots, x_{n}\right)$. Если же $n=2$, то выберем в классе $[\{0, f\}]_{A}$ такую функцию $h(x)$, что ее блоки отличны от множеств $\operatorname{Im}\left(a_{1} x_{1}^{2}+b_{1} x_{1}+c\right), \operatorname{Im}\left(a_{2} x_{2}^{2}+b_{2} x_{2}+c\right)$ и $\operatorname{Im} h$ не совпадает с блоками функции $a_{2} x_{2}^{2}+b_{2} x_{2}$. Тогда функция $f\left(x_{1}, h\left(f\left(x_{2}, x_{3}\right)\right)\right)$ возвращает нас к случаю $n \geqslant 3$. Лемма доказана.

Из теоремы 4 и леммы 10 вытекает следующее утверждение.

Теорема 5. Пусть $k \geqslant 4$. Всякий $A$-замкнутый класс функций из $P_{k}$, содержащий константы, совпадает с одним из классов, перечисленных в пn. 2-7 теоремы 4, либо (при $k=4)$ с одним из классов $L_{4}, L_{4} A_{4}, L_{4} V_{4}$.

Замечание 2. При $k \geqslant 4$ списки $A$-замкнутых и $S$-замкнутых классов функций из $P_{k}$, содержащих константы, совпадают (см. [11]).

Используя полученные выше утверждения об $A$-замкнутых классах линейных 
функций, содержащих константы, приведем полный список этих классов. Для упрощения записи функции, составляющие $A$-замкнутые классы, указываем с точностью до несущественных переменных.

$$
\begin{aligned}
& L_{4}^{(2)} S_{4}= L_{4} \cap S L P_{4}=Q L_{4}^{(2)+} \cup S_{4}=\left\{a_{1} x_{1}^{2}+b_{1} x_{1}+\ldots+a_{n} x_{n}^{2}+b_{n} x_{n}+c,\right. \\
&\left.a x^{2}+b x+c: n \geqslant 1, a_{1} b_{1}=\ldots=a_{n} b_{n} \neq 0\right\} ; \\
& L_{4}^{1(2)} S_{4}= L_{4} \cap P_{4}^{1}=F\left\langle\lambda_{1}(4)\right\rangle \cup S_{4}=\left\{a x^{2}+b x+c\right\} ; \\
& C S_{4}= F\left\langle\lambda_{2}(4)\right\rangle \cup S_{4}=\left\{a x^{2}+c, a x+c\right\} ; \\
& L_{4}^{(2)} A_{4}= Q L_{4}^{(2)+} \cup A_{4} \\
&=\left\{a_{1} x_{1}^{2}+b_{1} x_{1}+\ldots+a_{n} x_{n}^{2}+b_{n} x_{n}+c, b x+c: n \geqslant 1, a_{1} b_{1}=\ldots=a_{n} b_{n} \neq 0\right\} ; \\
& L_{4}^{1(2)} A_{4}= F\left\langle\lambda_{1}(4)\right\rangle \cup A_{4}=\left\{a x^{2}+b x+c, d x+c: a b \neq 0\right\} ; \\
& C A_{4}= F\left\langle\lambda_{2}(4)\right\rangle \cup A_{4}=\{b x+c\} ; \\
& L_{4}^{(2)} V_{4}= Q L_{4}^{(2)+} \cup V_{4} \\
&=\left\{a_{1} x_{1}^{2}+b_{1} x_{1}+\ldots+a_{n} x_{n}^{2}+b_{n} x_{n}+c, x+c: n \geqslant 1, a_{1} b_{1}=\ldots=a_{n} b_{n} \neq 0\right\} ; \\
& L_{4}^{1(2)} V_{4}= F\left\langle\lambda_{1}(4)\right\rangle \cup V_{4}=\left\{a x^{2}+b x+c, x+c: a b \neq 0\right\} ; \\
& C V_{4}= F\left\langle\lambda_{2}(4)\right\rangle \cup V_{4}=\{x+c, c\} ; \\
& L_{4}^{(2)} e_{4}= Q L_{4}^{(2)+} \cup e_{4} \\
&=\left\{a_{1} x_{1}^{2}+b_{1} x_{1}+\ldots+a_{n} x_{n}^{2}+b_{n} x_{n}+c, x, c: n \geqslant 1, a_{1} b_{1}=\ldots=a_{n} b_{n} \neq 0\right\} ; \\
& L_{4}^{1(2)} e_{4}= F\left\langle\lambda_{1}(4)\right\rangle \cup e_{4} \\
&=\left\{a x^{2}+b x+c, x, c: a b \neq 0\right\} ; \\
& C e_{4}= F\left\langle\lambda_{2}(4)\right\rangle \cup e_{4}=\{x, c\} ; \\
& L_{4}^{(2)}= Q L_{4}^{(2)+}=F\left\langle\lambda_{1}(4)\right\rangle=\left\{a x^{2}+b x+c, c: a b \neq 0\right\} ; \\
&= F\left\langle\lambda_{2}(4)\right\rangle=\{c\} \\
&\left.L_{1}^{2}+b_{1} x_{1}+\ldots+a_{n} x_{n}^{2}+b_{n} x_{n}+c, c: n \geqslant 1, a_{1} b_{1}=\ldots=a_{n} b_{n} \neq 0\right\} ; \\
&=
\end{aligned}
$$

где $\lambda_{1}(4)=(2,2), \lambda_{2}(4)=(4)$.

Взаимное расположение перечисленных выше классов показано на рис. 2.

\section{Список литературы}

1. Post E. L. Introduction to a general theory of elementary propositions. Amer. J. Math. (1921) 43, 163-185.

2. Post E. L. The Two-Valued Iterative Systems of Mathematical Logic. Princeton Univ. Press, Princeton, 1941.

3. Яблонский С. В., Гаврилов Г. П., Кудрявцев В. Б. Функции алгебры логики и классъ Поста. Наука, Москва, 1966.

4. Янов Ю. И., Мучник А. А. О существовании $k$-значных замкнутых классов, не имеющих конечного базиса. Докл. АН СССР (1959) 127, №1, 44-46. 
5. Марченков С. С. О замкнутых классах самодвойственных функций многозначной логики. Проблемъ кибернетики (1979) 36, 5-22.

6. Csákány B., Gavalcová T. Finite homogeneous algebras. I. Acta Sci. Math. (1980) 42, №1-2, 57-65.

7. Марченков С. С. Об однородных алгебрах. Докл. АН СССР (1981) 256, №4, 787-790.

8. Марченков С. С. Однородные алгебры. Проблемы кибернетики (1982) 39, 85-106.

9. Марченков С. С. О классификации алгебр со знакопеременной группой автоморфизмов. Докл. АН ССCP (1982) 265, №3, 533-536.

10. Нгуен Ван Хоа. О структуре самодвойственных замкнутых классов трехзначной логики $P_{3}$. Дискретная математика (1992) 4, №4, 82-95.

11. Нгуен Ван Хоа. О семействах замкнутых классов $k$-значной логики, сохраняемых всеми автоморфизмами. Дискретная математика (1993) 5, №4, 87-108.

12. Нгуен Ван Хоа. Описание замкнутых классов, сохраняемых всеми внутренними автоморфизмами $k$-значной логики. Докл. АН Беларуси (1994) 38, №3, 16-19.

13. Марченков С. С. Основные отношения $S$-классификации функций многозначной логики. Дискретная математика (1996) 8, №1, 99-128.

14. Марченков С. С. $S$-классификация идемпотентных алгебр с конечным носителем. Докл. РАН (1996) 348, №5, 587-589.

15. Марченков C. C. $G$-предполные классы многозначной логики. Дискретный анализ и исследование операций (1996) 3, №3, 47-70.

16. Марченков С. С. $S$-классификация функций многозначной логики. Дискретная математика (1997) 9, №3, 125-152.

17. Нгуен Ван Хоа. О структуре замкнутых классов $k$-значной логики, самодвойственных относительно транзитивных групп. Докл. АН Беларуси (1994) 38, №6, 17-20.

18. Нгуен Ван Хоа. О замкнутых классах $k$-значной логики, самодвойственных относительно транзитивных групп. Дискретная математика (1996), 8, №1, 129-156.

19. Марченков С. С. О замкнутых классах самодвойственных функций многозначной логики. II. Проблемы кибернетики (1983) 40, 261-266.

20. Rosenberg I. G. Über die funktionale Vollständigkeit in den mehrwertigen Logiken. Rozpravy Československe Akad. Věd. Ǩada Mat. Přirod. Věd. (1970) 80, 3-93.

21 . Бурле Г. А. Классы $k$-значной логики, содержащие все функции одной переменной. Дискретный анализ (1967) 10, 3-7.

22. Яблонский С. В. Введение в теорию функций $k$-значной логики. Дискретная математика и математические вопросы кибернетики. Наука, Москва, 1974, 9-66.

23. Яблонский С. В. Введение в дискретную математику. Наука, Москва, 1986.

24. Каргаполов М. И., Мерзляков Ю. И. Основы теории групп. Наука, Москва, 1972.

25. Мальцев А. И. Итеративные алгебры Поста. Изд. НГУ, Новосибирск, 1976.

Статья поступила 28.08.1997. Переработанный вариант поступил 28.01.1998. 